\title{
EFEITO DA FRAÇÃO ACETATO DE ETILA EXTRAÍDA DAS FOLHAS DE EUGENIA INVOLUCRATA (MYRTACEAE) SOBRE A ATIVIDADE DA ACETILCOLINESTERAS E BUTIRILCOLINESTERASE EM RATOS DIABÉTICOS
}

\author{
EFFECT OF THE ETHYL ACETATE FRACTION EXTRACTED FROM THE LEAVES \\ OF EUGENIA INVOLUCRATA (MYRTACEAE) ON THE ACTIVITY OF ACETYL \\ CHOLINESTERASE AND BUTYL CHOLINESTERASE IN DIABETIC RATS
}

Hediana Grazielly dos Passos ${ }^{5}$, Harrison Gustavo Pech Corrêa', Sheila Wayszceyk', Daniela Delwing
de Lima', Michele Debiasi Alberton', Sara Cristiane Barauna, Cláudia Almeida de Coelho
Albuquerque ${ }^{3}$ e Débora Delwing Dal Magro ${ }^{3 *}$
'Departamento de Medicina, Universidade Regional de Blumenau, Blumenau, SC, Brasil.

2Programa de Pós-graduação em Saúde e Meio Ambiente, Universidade da Região de Joinville- UNIVILLE, Joinville, SC, Brasil.

${ }^{3}$ Departamento de Ciências Naturais, Universidade Regional de Blumenau, Blumenau, SC, Brasil.

${ }^{4}$ Departamento de Farmácia, Universidade Regional de Blumenau, Blumenau, SC, Brasil.

${ }^{5}$ Departamento de Biomedicina, Universidade Regional de Blumenau, Blumenau, SC, Brasil.

*Autor correspondente: deboradelwing@furb.br Departamento de Ciências Naturais, Universidade Regional de Blumenau, Rua Antônio da Veiga, 140, CEP 89012-900, Blumenau, SC, Brasil. Telefone: 554733210272

\section{RESUMO}

Durante a hiperglicemia persistente, ocorre um aumento da produção de radicais livres de oxigênio através da auto oxidação da glicose, causando estresse oxidativo. A Doença de Alzheimer está associada com a diminuição de diversos neurotransmissores cerebrais, como a acetilcolina, a noradrenalina e a serotonina. Considerando que a diabetes persistente causa hiperglicemia, levando ao aumento na produção de radicais livres, que os radicais livres podem contribuir com o processo de neurodegeneração e que Eugenia involucrata possui compostos fenólicos e flavonoides que agem como antioxidantes, o objetivo deste trabalho foi verificar os efeitos da administração crônica e aguda da fração acetato de etila (FAE), obtida das folhas da espécie Eugenia involucrata, sobre os efeitos causados pela diabetes na atividade da acetilcolinesterase (AChE) em cérebro e butirilcolinesterase (BUChE) em sangue de ratos. Para tanto, ratos Wistar, de 60 dias, foram divididos em 09 grupos, sendo grupo Controle, grupo Rivastigmina, grupo FAE (50, 100 e 150mg/kg), grupo FAE (50, 100 e 150 mg/kg) + Diabetes e grupo Diabético. Após 12 horas do último tratamento (agudo e crônico), os animais foram sacrificados. O cérebro foi removido para determinação da atividade da AChE e o sangue coletado para determinação da atividade da BuChE. No tratamento agudo e crônico, a FAE inibiu a atividade da AChE em Córtex Cerebral e em Hipocampo ao se comparar com o grupo controle. Em relação a atividade da BuChE, no tratamento crônico, a FAE inibiu a atividade da enzima quando comparado com o grupo controle.

Palavras-Chave: Eugenia involucrata, colinesterases, diabetes, estresse oxidativo. 


\section{ABSTRACT}

During persistent hyperglycemia, oxygen free radical production increases through glucose self-oxidation, causing oxidative stress. Alzheimer's Disease is associated with decreased brain neurotransmitters, such as acetylcholine, norepinephrine, and serotonin. Considering that persistent diabetes causes hyperglycemia leading to increased free radical production, that free radicals may contribute to the neurodegeneration process and that Eugenia involucrata has phenolic and flavonoid compounds acting as antioxidants, the aim of this study was to verify the effects of chronic and acute administration of the ethyl acetate fraction (FAE) obtained from the leaves of the species Eugenia involucrata on the effects caused by diabetes on the activity of acetylcholinesterase (AChE) in brain and butyrylcholinesterase (BUChE) in rat blood. Therefore, 60-day-old Wistar rats were divided into 9 groups: Control group, Rivastigmine group, FAE group (50, 100 and $150 \mathrm{mg} / \mathrm{kg})$, FAE group $(50,100$ and $150 \mathrm{mg} / \mathrm{kg})+$ Diabetes and Diabetic group. 12 hours after the last treatment (acute and chronic), the animals were sacrificed by decapitation in the absence of anesthesia. The brain was removed for AChE activity determination and blood collected for BUChE activity determination. In acute and chronic treatment, FAE inhibited AChE activity in the cerebral cortex and hippocampus when compared with the control group. Regarding BuChE activity in chronic treatment, FAE inhibited enzyme activity when compared to the control group. Thus, it is suggested that FAE has anticholinesterase action.

Keywords: Eugenia involucrata, cholinesterases, diabetes, oxidative stress.

\section{INTRODUÇÃO}

Registros em literatura constam que a doença Diabetes Mellitus (DM) é considerada uma epidemia de caráter mundial, ou seja, um problema de prevalência elevada e incidência crescente. A doença está diretamente relacionada com a qualidade de vida dos portadores, sendo esta traduzida por uma dieta inadequada, pelo sedentarismo, pela obesidade, pelo envelhecimento da população e pela urbanização crescente, vinculada às complicações crônicas que pioram a expectativa de vida dos diabéticos, sendo um problema para os sistemas de saúde responsáveis (BRASIL, 2006; ROCHA et al., 2006; SILVA; OLIVEIRA; NAGEM, 2010; AMORIM; BURGOS; CABRAL, 2017)

Sendo assim, a DM é referida como transtorno metabólico de etiologia múltipla, caracterizada pela hiperglicemia, dislipidemias e obesidade, resultantes de distúrbios na secreção e/ou na ação da insulina. A hiperglicemia crônica associa-se em longo prazo com danos, insuficiências e disfunções de vários órgãos, entre eles, olhos, rins, coração e vasos sanguíneos, além do sistema nervoso central e periférico, manifestando alterações no metabolismo de lipídios, proteínas e carboidratos (SILVA; OLIVEIRA; NAGEM, 2010; BRASIL, 2013; CIPRIANI, 2013; BRAGA et al., 2017; BRINATI et al., 2017)

Em relação à classificação da DM, pode ser dividida em "tipo 1" (DM1) e "tipo 2" (DM2), ou seja, enquanto na DM1 não há produção de insulina pelo pâncreas, na DM2 essa produção é relativamente deficiente, caracterizada pela insulina diminuir sua capacidade de estimular a utilização da glicose, tendo como causa uma deficiência no receptor da insulina ou, então, um defeito específico no mecanismo pós-receptor quando utilizado (BRASIL, 2006; SERON; FURLAN, 2010).

Salienta-se que na doença há aumento da produção de radicais livres, que são as substâncias que estimulam alterações celulares. Múltiplas complicações estão associadas a produção de radicais livres, seja em excesso e/ou à redução dos mecanismos antioxidantes endógenos. As complicações 
oxidativas induzidas nas células e tecidos, junto com o aumento da produção de radicais livres na DM, tem papel importante quando se trata de implicações na etiologia de diversas doenças degenerativas comuns (SERON; FURLAN, 2010). A enzima acetilcolinestersase (AChE) contribui para a integridade e permeabilidade da membrana sináptica durante a neurotransmissão e condução do impulso nervoso (GRAFIUS et al., 1971). Estudos relatam que a atividade da AChE é diminuída pela ação de radicais livres e que a administração de antioxidantes previne esta redução (TSAKIRIS et al., 2000; MELO et al., 2003). De acordo com a literatura, pacientes com Doença de Alzheimer apresentam redução na atividade da AChE em córtex cerebral e hipocampo (FISHMAN et al., 1986), e alterações na atividade da AChE estão associadas às alterações cognitivas características desses pacientes.

A introdução do processo de estresse oxidativo procede da existência de um desequilíbrio entre os compostos oxidantes e antioxidantes em relação à excessiva produção de radicais livres ou perda da capacidade de remoção destes. Então, na hiperglicemia persistente ocorre uma elevação da produção de radicais livres de oxigênio pela oxidação da glicose, levando ao estresse oxidativo (MAZZANTI et al., 2003; ROCHA et al., 2006; BARBOSA et al., 2010; MOREIRA; BOAS; FERREIRA, 2014; FRANCISQUETI et al., 2017). O déficit cognitivo, presente em pacientes com Doença de Alzheimer, também poderá ocorrer em portadores de DM. Ambas as patologias estão associadas ao estresse oxidativo, o qual supõe-se estar envolvido na fisiopatologia destas doenças (CUMMINGS, 2000; LAW; GAUTHIER; QUIRION, 2001; PETRONILHO, PINTO \& VILLAR et al., 2011).

A Eugenia involucrata (Myrtaceae), mais conhecida como cerejeira ou cerejeira-do-mato, encontrada no Sul do Brasil, possui alto potencial antioxidante pela rica presença de compostos fenólicos (CAMILA LEANDRA BUENO DE ALMEIDA, 2013; RODRIGUES DA SILVA et al., 2015; CIPRIANI, 2016). Dessa forma, considerando que a diabetes persistente causa hiperglicemia, levando ao aumento na produção de radicais livres, que os radicais livres podem contribuir com o processo de neurodegeneração, que Eugenia involucrata possui compostos fenólicos e flavonoides que agem como antioxidantes e que estudos in vitro mostraram ação anticolinesterásica para a fração acetato de etila (FAE), obtida das folhas da espécie Eugenia involucrata, o objetivo deste trabalho foi verificar os efeitos da administração crônica e aguda da FAE, obtida das folhas da espécie Eugenia involucrata, sobre os efeitos causados pela diabetes na atividade da acetilcolinesterase (AChE) em cérebro e butirilcolinesterase (BuChE) em sangue de ratos (DALLAQUA; DAMASCENO, 2011; FERREIRA et al., 2011; DOS SANTOS et al., 2014).

\section{MATERIAIS E MÉTODOS}

\section{MATERIAL VEGETAL}

As partes aéreas da espécie Eugenia involucrata foram coletadas na cidade de Rodeio-SC (265'22" S; 49²1'59" W), em abril de 2014. O material vegetal foi identificado pelos botânicos Marcos Sobral (Universidade Federal de São João Del-Rey/MG) e André Luis de Gasper (Fundação Universidade Regional de Blumenau - FURB). A exsicata está catalogada no Herbário Roberto Miguel Klein, da Universidade Regional de Blumenau, sob o registro FURB 42884. As partes aéreas obtidas foram separadas em folhas e galhos, sendo a porção das folhas destinada à obtenção do extrato bruto hidroalcoólico (CIPRIANI, 2016). 


\section{PREPARO DO EXTRATO BRUTO HIDROALCOÓLICO (EBH)}

Após a coleta e identificação, o material vegetal foi mantido à temperatura ambiente até peso constante e moído em moinho de facas. Este material foi submetido ao procedimento de maceração em etanol 70\% durante 7 dias, sendo este processo repetido mais uma vez. O extrato obtido foi filtrado em funil de Büchner e concentrado em evaporador rotatório sob pressão reduzida em banho-maria com temperatura controlada de $50^{\circ} \mathrm{C}$ durante todo o procedimento, até completa secagem. O rendimento foi calculado em porcentagem em relação à quantidade de material seco utilizado para a extração (CIPRIANI, 2016).

\section{FRACIONAMENTO DO EBH}

Após macerado e concentrado, uma alíquota de $300 \mathrm{~g}$ do extrato bruto hidroalcoólico foi ressuspendida em solução hidroalcoólica $10 \%$ e mantida em repouso em geladeira por 24 horas (2 a $8^{\circ} \mathrm{C}$ ) para decantação. Este processo resultou em um resíduo insolúvel que foi separado por meio de filtração à vácuo. O sobrenadante foi fracionado através de partição líquido-líquido em funil de separação com solventes de polaridades distintas, onde foi obtida a fração acetato de etila (FAE). As frações obtidas foram evaporadas em evaporador rotatório e, após completa secagem, tiveram suas massas determinadas e o percentual de rendimento calculado com base na quantidade de material vegetal utilizado para a partição. A FAE (60 g) foi encaminhada aos ensaios biológicos (CIPRIANI, 2016).

\section{ANIMAIS E TRATAMENTO}

Ratos da linhagem Wistar, com idade aproximada de 60 dias, provenientes do Biotério Central da FURB, foram utilizados. Os animais foram desmamados aos 21 dias de idade e após, mantidos em um ciclo de 12 horas claro/escuro, à temperatura constante de $22^{\circ} \mathrm{C}$ e com livre acesso à comida e água. Os cuidados com os animais seguiram as normas aprovadas pelo Comitê de Ética da Universidade Regional de Blumenau (protocolo:16/2016).

Os animais foram divididos em 09 grupos, sendo grupo Controle -, que recebeu injeção intraperitoneal de $\mathrm{NaCl} 0,9 \%$ e água via gavagem; grupo Controle + , que recebeu injeção intraperitoneal de $\mathrm{NaCl}$ 0,9\% e Rivastigmina $1,5 \mathrm{mg} / \mathrm{Kg}$ no tratamento agudo e $0,5 \mathrm{mg} / \mathrm{Kg}$ no tratamento crônico via gavagem; grupo tratamento-FAE, obtido das folhas da espécie Eugenia involucrata, que receberam injeção intraperitoneal de $\mathrm{NaCl} 0,9 \%$ e FAE $(50 \mathrm{mg} / \mathrm{kg}, 100 \mathrm{mg} / \mathrm{kg}$ e $150 \mathrm{mg} / \mathrm{kg})$ via gavagem; grupo tratamento-FAE+diabetes, obtido das folhas da espécie Eugenia involucrata, que receberam injeção intraperitoneal de aloxana (150mg/Kg) e FAE $(50 \mathrm{mg} / \mathrm{kg}, 100 \mathrm{mg} / \mathrm{kg}$ e $150 \mathrm{mg} / \mathrm{kg}$ ) via gavagem; grupo Diabético, que recebeu injeção intraperitoneal de aloxana (150mg/Kg) e água via gavagem. Destes animais, uma parte $(1 / 2)$ foi submetida a um tratamento (crônico, 15 dias) que teve início após a indução da diabetes, e a outra parte $(1 / 2)$ dos animais foi submetida a um tratamento agudo, realizado após indução da diabetes. Para o tratamento agudo, estes foram tratados uma única vez após a confirmação da diabetes. 12 horas após o último tratamento (agudo e crônico), os animais foram sacrificados por decapitação na ausência de anestesia e, em seguida, o cérebro foi removido para determinação da atividade da AChE e o sangue coletado para determinação da atividade da BuChE. Foi utilizado o fármaco Rivastigmina, do fabricante Legrand, como controle positivo. 


\section{INDUÇÃO DE DM}

Para a indução da DM, foi administrado aloxana (diluído em solução salina) na dose de 150mg/ $\mathrm{kg}$, via intraperitoneal, dose única, após um período de jejum de 24 horas. Após 6 horas da indução, foi fornecida uma solução de glicose $10 \%$ como única fonte hídrica durante 24 horas para evitar uma hipoglicemia fatal devido à liberação maciça de insulina que ocorre após a destruição das células §. Após a administração da aloxana, a glicose periférica foi determinada e os animais que apresentaram uma glicemia casual inferior a $200 \mathrm{mg} / \mathrm{dL}$ foram novamente induzidos, seguindo o mesmo protocolo. Somente os animais com glicemia casual superior a $200 \mathrm{mg} / \mathrm{dl}$ foram considerados diabéticos e selecionados para o experimento (MAZZANTI et al., 2003; LEHMANN et al., 2015).

\section{ESTUDOS BIOQUÍMICOS}

\section{Preparo das amostras}

Após a dissecação, o cérebro e o sangue foram rapidamente removidos. O hipocampo e o córtex cerebral foram homogeneizados em tampão fosfato de potássio, $\mathrm{pH}$ 7,5. O homogeneizado foi centrifugado a $1000 \mathrm{x}$ g por $10 \mathrm{~min}$, o pellet foi descartado e o sobrenadante foi utilizado para a determinação da atividade da AChE e concentração proteica. Após coletado, o sangue foi rapidamente centrifugado a 1000 x g por 10 min e o soro foi separado e utilizado para a determinação da atividade da BuChE e concentração proteica.

\section{Ensaio de atividade AChE}

A atividade da acetilcolinesterase foi determinada de acordo com Ellman et al. (1961) com algumas modificações. A taxa de hidrólise foi medida em concentração de acetilcolina de $0,8 \mathrm{mM}$ em soluções de ensaio de $1 \mathrm{~mL}$ com tampão fosfato $30 \mathrm{mM}$, pH 7,5 e 5,5'-Ditiobis- (ácido 2-nitrobenzóico) 1,0 mM (DTNB) a $25^{\circ} \mathrm{C} .50 \mu \mathrm{l}$ de sobrenadante de hipocampo ou córtex cerebral de rato foram adicionados à mistura e pré-incubados por 3 min. A hidrólise foi monitorada pela formação do diânion de tiolato de DTNB a 412 nm durante 2-3 min (intervalos de $30 \mathrm{~s}$ ). Todas as amostras foram testadas em duplicata. Os resultados foram expressos em umol/ACSCh/h/mg proteína.

\section{Ensaio de atividade BuChE}

A atividade da butirilcolinesterase foi determinada pelo método de Ellman et al. (1961) com algumas modificações. A taxa de hidrólise foi medida em concentração de acetiltiocolina de 0,8 mM em soluções de ensaio de $1 \mathrm{~mL}$ com tampão fosfato $100 \mathrm{mM}$, pH 7,5 e DTNB 1,0 mM. $50 \mu \mathrm{l}$ de soro de rato foram adicionados à mistura e pré-incubados por $3 \mathrm{~min}$. A hidrólise foi monitorada pela formação do diânion de tiolato de DTNB a $412 \mathrm{~nm}$ por 2-3 min (intervalos de $30 \mathrm{~s}$ ). Todas as amostras foram testadas em duplicata. Os resultados foram expressos em umol/ACSCh/h/mg proteína.

\section{Dosagem de proteínas}

A determinação das proteínas foi realizada pelo método de Bradford e colaboradores (1976), utilizando albumina sérica bovina como padrão (BRADFORD, 1976). 


\section{ANÁLISE ESTATÍSTICA}

Os resultados nos diferentes grupos foram representados como média \pm desvio padrão, analisados através do Programa SPSS para Windows, versão 12 (SSPS, Chicago, IL, USA), utilizando-se a análise de variância (ANOVA), seguida do teste post-hoc de Duncan para comparação entre as médias dos grupos, sendo que os valores de $p<0,05$ foram considerados significativos.

\section{RESULTADOS}

No que diz respeito ao tratamento agudo, o grupo diabético não alterou a atividade da AChE em hipocampo (A) e córtex cerebral (B), bem como a atividade da BuChE (C) em soro de ratos. A Rivastigmina e a FAE, em todas as doses testadas $(50 \mathrm{mg} / \mathrm{Kg}, 100 \mathrm{mg} / \mathrm{Kg}$ e $150 \mathrm{mg} / \mathrm{Kg}$ ), diminuíram a atividade da AChE em hipocampo (F5,30=21.955; $\mathrm{p}<0.001)$ e córtex cerebral $(\mathrm{F} 5,30=22.718 ; \mathrm{p}<0.001)$ quando comparado ao grupo Controle. Em soro, a rivastigmina diminuiu a atividade da BuChE, porém a FAE não alterou atividade da enzima (F5,26=16.797; $\mathrm{p}<0.001)$ (Figura 1).

Figura 1- Efeito da diabetes induzida por aloxana e da administração aguda de rivastigmina e da fração acetato de etila, obtida das folhas de Eugenia involucrata $(50 \mathrm{mg} / \mathrm{Kg}, 100 \mathrm{mg} / \mathrm{Kg}, 150 \mathrm{mg} /$ $\mathrm{Kg}$ ) sobre a atividade da AChE em hipocampo (A) e córtex cerebral (B), e sobre a atividade da BuChE (C) em soro de ratos. Os dados representam a média \pm desvio padrão de 6-7, experimentos independentes (animais) realizados em duplicata. Diferente do controle, ${ }^{* *} p<0.001$ (Teste Múltiplo de Duncan). FAE: fração acetato de etila.

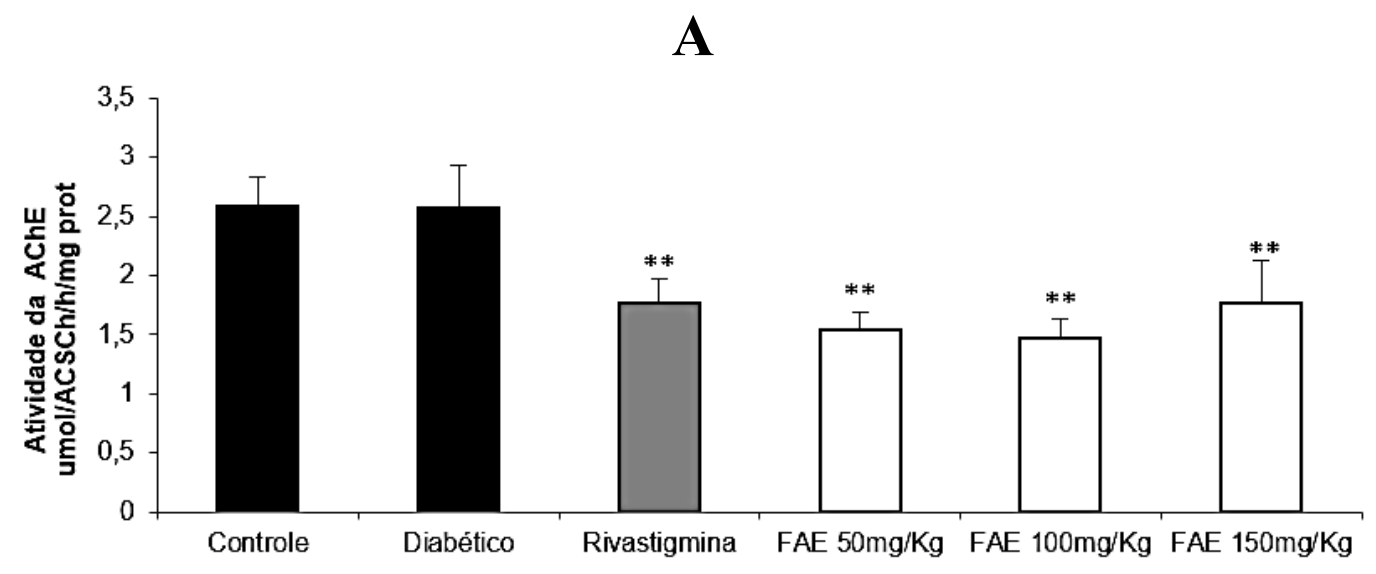

B

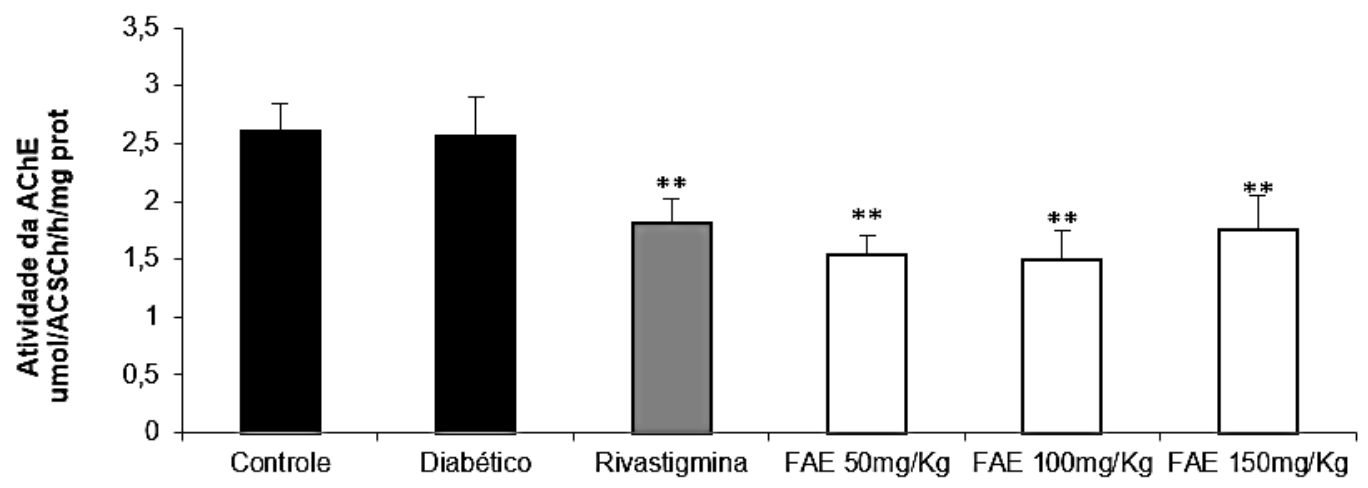


C

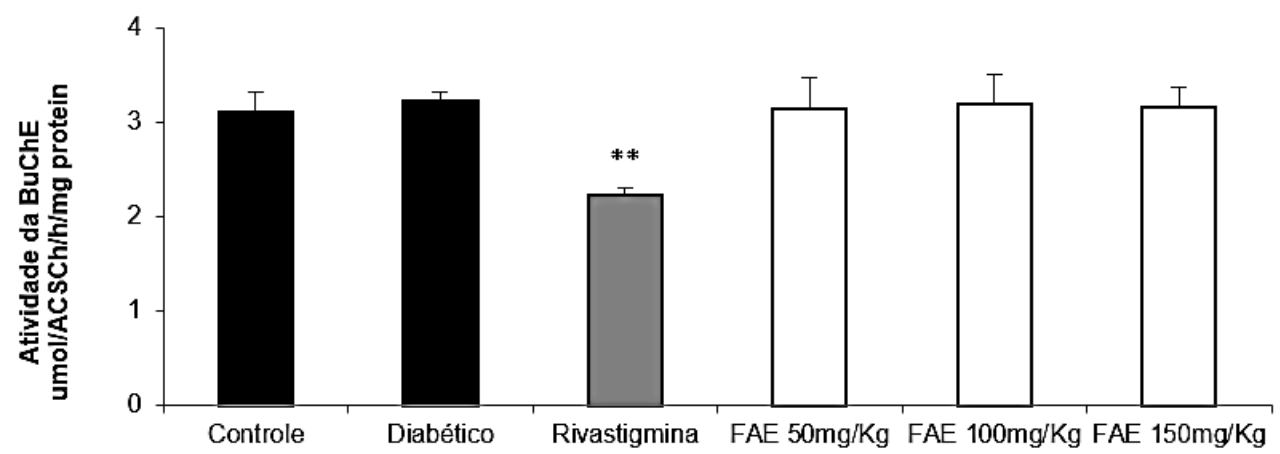

No tratamento crônico, o grupo diabético não alterou a atividade da AChE em hipocampo (A) e córtex cerebral (B), bem como a atividade da $\mathrm{BuChE}(\mathrm{C})$ em soro de ratos. A Rivastigmina diminuiu a atividade da AChE em hipocampo e córtex cerebral, e a atividade da BuChE em soro de ratos quando comparada ao grupo controle. A FAE nas doses 50mg/Kg, 100mg/Kg e 150mg/Kg diminuiu a atividade da AChE em hipocampo $\left(\mathrm{F}_{5,28}=16.569 ; \mathrm{p}<0.001\right)$ e a atividade da BuChE em soro $\left(\mathrm{F}_{5,27}=24.489 ; \mathrm{p}<0.001\right) \mathrm{de}$ ratos quando comparados ao grupo controle. Em córtex cerebral, a FAE, nas doses de 100 e 150mg/ $\mathrm{Kg}$, diminuiu a atividade da $\mathrm{AChE}\left(\mathrm{F}_{5,26}=21.443 ; \mathrm{p}<0.001\right)$ quando comparada ao grupo controle.

Figura 2 - Efeito da diabetes induzida por aloxana, da administração crônica de rivastigmina e da fração acetato de etila, obtida das folhas de Eugenia involucrata $(50 \mathrm{mg} / \mathrm{Kg}, 100 \mathrm{mg} / \mathrm{Kg}, 150 \mathrm{mg} /$ $\mathrm{Kg}$ ) sobre a atividade da AChE em hipocampo (A) e córtex cerebral (B) e sobre a atividade da BuChE (C) em soro de ratos. Os dados representam a média \pm desvio padrão de 6-7 experimentos independentes (animais) realizados em duplicata. Diferente do controle, ${ }^{* *} p<0.001$ (Teste Múltiplo de Duncan). FAE: fração acetato de etila.

A

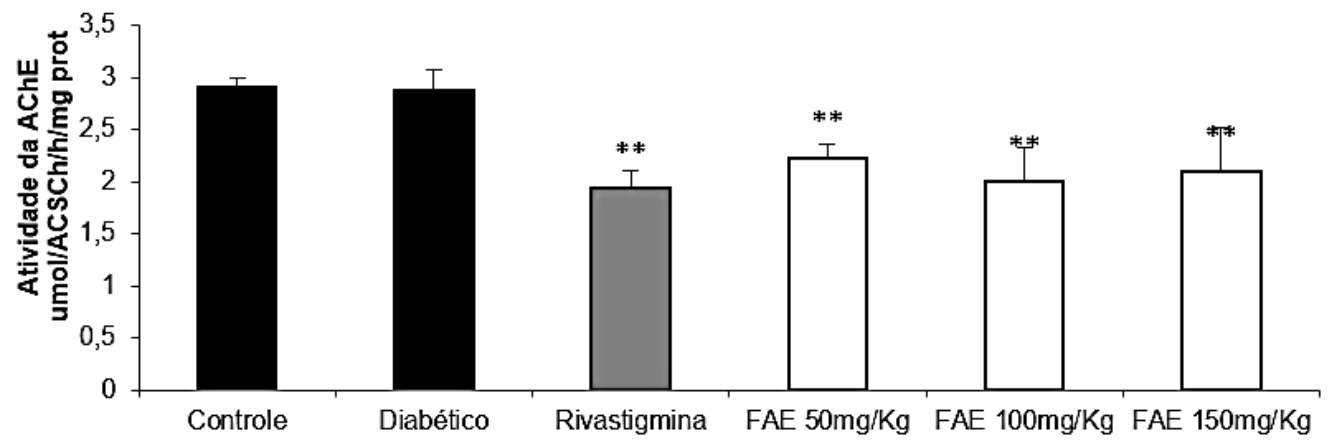

B

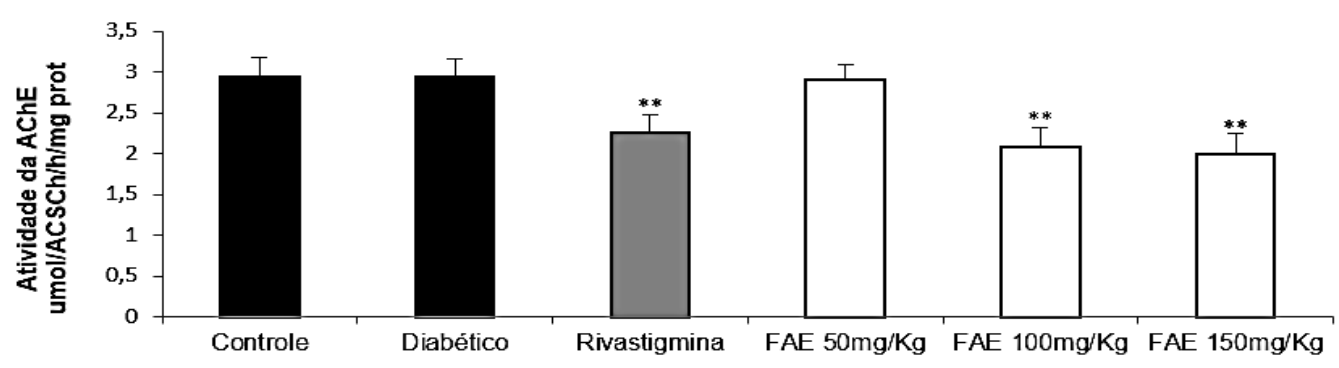




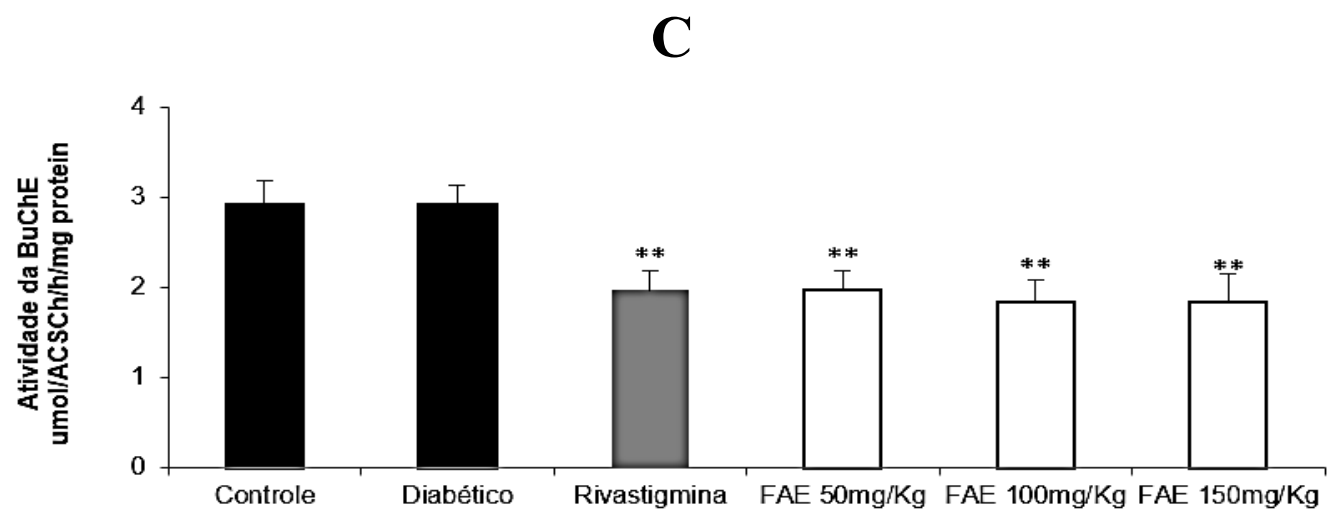

\section{DISCUSSÃO}

A DM pode ser entendida como um grupo de doenças metabólicas caracterizada pelo aumento nos níveis de glicose sanguínea devido a um distúrbio na secreção e/ou na ação da insulina. Essa doença tem sido considerada como uma epidemia, um dos maiores problemas de saúde a ser resolvido em todo mundo. Estudos epidemiológicos mostram que a prevalência e a incidência da diabetes no Brasil têm aumentado a cada ano. Estima-se que existiam 11,9 milhões de indivíduos vivendo com diabetes no Brasil em 2014 (IDF, 2014). A urbanização crescente, o estilo de vida pouco saudável, que inclui dieta com alto teor de gordura e diminuição da atividade física, obesidade e o envelhecimento populacional, são fatores responsáveis pela tendência crescente da incidência da doença na população, gerando um elevado gasto do poder público para o controle e tratamento de suas complicações (WILD et al., 2004).

A doença apresenta como principal característica a hiperglicemia, devido a distúrbios no metabolismo de lipídeos, carboidratos e proteínas (SILVA; COSTA, 2008; FERREIRA et al., 2011). A exposição prolongada dos tecidos à hiperglicemia causa danos endoteliais, cujos principais mecanismos envolvidos são o aumento da atividade da via dos poliois, a glicação não-enzimática das proteínas, o estresse oxidativo, com o aumento dos radicais livres, e as alterações da proteína quinase C (SCHALKWIJK; STEHOUWER, 2005). Uma das causas das complicações da diabetes é o estresse oxidativo, condição na qual as defesas antioxidantes não são suficientes para inativar as espécies reativas geradas. A doença está associada a distúrbios metabólicos progressivos e disfunção em diferentes órgãos como o pâncreas, fígado, coração, rins, nervos e outros (HASHEMPUR et al., 2015). O déficit cognitivo, presente em pacientes com Doença de Alzheimer, também poderá ocorrer em portadores de DM. Ambas as patologias estão associadas ao estresse oxidativo, o qual supõe-se estar envolvido na fisiopatologia destas doenças (CUMMINGS, 2000; LAW; GAUTHIER; QUIRION, 2001; PETRONILHO, PINTO \& VILLAR et al., 2011).

Considerando que a diabetes persistente causa hiperglicemia, levando ao aumento na produção de radicais livres, que os radicais livres podem contribuir com o processo de neurodegeneração e que Cipriani (2016) demonstrou, em um de seus estudos, que a FAE de Eugenia involucrata tem atividade anticolinesterásica in vitro, neste estudo, verificamos os efeitos da administração aguda e crônica (in vivo) da FAE sobre a atividade das enzimas AChE e BuChE em cérebro e soro de ratos da linhagem Wistar, respectivamente. 
A AChE é uma das enzimas de maior importância e necessidade no funcionamento do sistema nervoso central. O aumento da atividade da enzima pode prejudicar a formação da memória no hipocampo e o processo de transmissão sináptica. No que diz respeito ao tratamento agudo, o grupo diabético não alterou a atividade da $\mathrm{AChE}$ em hipocampo e córtex cerebral, bem como a atividade da BuChE em soro de ratos. A Rivastigmina e a FAE, em todas as doses testadas (50mg/Kg, 100mg/Kg e $150 \mathrm{mg} / \mathrm{Kg}$ ), diminuiu a atividade da $\mathrm{AChE}$ em hipocampo e córtex cerebral. Em soro, a rivastigmina diminuiu a atividade da BuChE, porém a FAE não alterou atividade da enzima. No tratamento crônico, o grupo diabético não alterou a atividade da AChE em hipocampo e córtex cerebral, bem como a atividade da BuChE em soro de ratos. A Rivastigmina diminuiu a atividade da AChE em hipocampo e córtex cerebral e a atividade da BuChE em soro de ratos. A FAE, nas doses $50 \mathrm{mg} / \mathrm{Kg}, 100 \mathrm{mg} / \mathrm{Kg}$ e $150 \mathrm{mg} / \mathrm{Kg}$, diminuiu a atividade da AChE em hipocampo e a atividade da BuChE em soro de ratos quando comparados ao grupo controle. Em córtex cerebral, a FAE, nas doses de 100 e $150 \mathrm{mg} /$ $\mathrm{Kg}$, diminuiu a atividade da AChE. Nossos resultados sugerem que a FAE, ao diminuir a atividade da AChE e BuChE, diminui a degradação da acetilcolina, prolongando sua ação na fenda sináptica. Na terapêutica, a estratégia farmacológica prevalente no tratamento da DA, consiste na utilização dos inibidores da AChE, com o objetivo de aumentar a concentração de acetilcolina na fenda sináptica e, consequentemente, melhorar a neurotransmissão colinérgica no sistema nervoso central (SNC) (COYLE; KERSHAW, 2001). O uso de inibidores da AChE melhora consideravelmente os sintomas da DA e estabiliza o declínio das habilidades funcionais e das funções cognitivas.

A perda de memória é relatada na DM (ALMEIDA-PITITTO; ALMADA FILHO; CENDOROGLO, 2008), mas, em nosso trabalho, o grupo diabético não apresentou alteração na atividade da AChE. O motivo da AChE não ter sido alterada pode estar relacionado a duração do tratamento, uma vez que mesmo no tratamento crônico, onde os animais foram tratados por 15 dias, não houve alteração na atividade da enzima. Pretendemos, no futuro, prolongar a duração do tratamento crônico, com o intuito de verificar uma possível alteração a longo prazo. As enzimas colinesterases estão presentes em abundância no sistema nervoso central e têm sido foco de intensa investigação por causa do envolvimento na transmissão colinérgica, das consequências deletérias de sua inibição e da sua possível contribuição à patologia observada em alguns distúrbios do sistema nervoso central, como sua participação na Doença de Alzheimer (GEULA et al., 1995; DARVESH, HOPKINS \& GEULA, 2003). Além disso, a literatura relata que há algumas evidências mostrando que além da AChE, a BuChE também hidrolisa a acetilcolina (MESULAM et al., 2002). Corroborando com nossos resultados, Silveira e colaboradores (2011), mostraram em seu estudo a atividade anticolinesterásica dos frutos de Myrcianthes pungens, pertencente à mesma família da Eugenia involucrata, MYRTACEAE. Lima (2016), através de compostos isolados de Myrciaria ferruginea, também verificou diminuição da atividade da AChE.

\section{CONCLUSÃO}

Concluindo, sugere-se que a FAE, obtida das folhas de Eugenia involucrata, nas doses analisadas, apresenta atividade anticolinesterásica, uma vez que diminuiu a atividade das enzimas AChE e BuChE em cérebro e soro de ratos, respectivamente. Em nossos estudos, a FAE foi tão eficaz quanto a Rivastigmina em diminuir a atividade das enzimas. Dessa forma, novos estudos são necessários para identificação, quantificação e isolamento de compostos bioativos, a fim de darmos continuidade aos nossos estudos. 
EFEITO DA FRAÇÃo ACETATO DE ETILA EXTRAÍDA DAS FOLHAS DE EUGENIA INVOLUCRATA (MYRTACEAE) SOBRE A ATIVIDADE DA...

\section{REFERÊNCIAS}

ALMEIDA-PITITTO, B. de; ALMADA FILHO, C. de M.; CENDOROGLO, M. S. Déficit cognitivo: mais uma complicação do diabetes melito? Arquivos Brasileiros de Endocrinologia \& Metabologia, v. 52, n. 7 , p. 1076-1083, 2008. Disponível em: <https://www.scielo.br/pdf/abem/v52n7/03.pdf>. Acesso em: 08 nov. 2017.

AMORIM, T. C. de; BURGOS, M. G. P. de A.; CABRAL, P. C. Perfil clínico e antropométrico de pacientes idosos com diabetes mellitus tipo 2 atendidos em ambulatório. Scientia Medica, v. 27, n. 3, p. 1-5, 2017. Disponível em: <https://www.researchgate.net/publication/318669348_Perfil_ clinico_e_antropometrico_de_pacientes_idosos_com_diabetes_mellitus_tipo_2_atendidos_em_ ambulatorio>. Acesso em: 06 nov. 2017.

BARBOSA, K. B. F. et al. Estresse oxidativo: Conceito, implicações e fatores modulatórios. Revista de Nutricao, v. 23, n. 4, p. 629-643, 2010. Disponível em: <https://www.scielo.br/scielo.php?script=sci_ arttext\&pid=S1415-52732010000400013>. Acesso em: 06 nov. 2017.

BRADFORD, M. M. A rapid and sensitive method for the quantitation of microgram quantities of protein utilizing the principle of protein-dye binding. Anal. Biochem., v. 72, p. 248, 1976. Disponível em: <https://pubmed.ncbi.nlm.nih.gov/942051/>. Acesso em: 10 nov. 2017.

BRASIL. Ministério da Saúde. Secretaria de Atenção à Saúde. Departamento de Atenção Básica. Diabetes Mellitus / Ministério da Saúde. Secretaria de Atenção à Saúde, Departamento de Atenção Básica - Brasília: Ministério da Saúde, 2006. 56p. Disponível em: < https://bvsms.saude.gov. br/bvs/publicacoes/diabetes_mellitus_cab16.pdf>. Acesso em: 07 nov. 2017.

BRASIL. Ministério da Saúde. Secretaria de Atenção à Saúde. Departamento de Atenção Básica. Estratégias para o cuidado da pessoa com doença crônica: diabetes mellitus / Ministério da Saúde, Secretaria de Atenção à Saúde, Departamento de Atenção Básica. - Brasília: Ministério da Saúde, 2013. 160 p. Disponível em: < https://bvsms.saude.gov.br/bvs/publicacoes/estrategias_ cuidado_pessoa_diabetes_mellitus_cab36.pdf>. Acesso em: 07 nov. 2017.

BRASIL. Ministério da Saúde. Secretaria de Atenção à Saúde. Departamento de Atenção Básica. Protocolo Clínico e Diretrizes Terapêuticas: Doença de Alzheimer. Secretaria de Atenção à Saúde, Departamento de Atenção Básica - Brasília: Ministério da Saúde, 2013. 22 p. Disponível em: < https://portalarquivos2.saude.gov.br/images/pdf/2017/dezembro/08/465660-17-10-MINUTA-dePortaria-Conjunta-PCDT-Alzheimer-27-1 1-2017---COMPLETA.pdf>. Acesso em: 07 nov. 2017.

BRAGA, D. C. et al. Fatores associados à depressão em indivíduos com diabetes mellitus. Arquivos Catarinenses de Medicina, v. 46, n. 3, p. 118-128, 2017. Disponível em: <http://www.acm.org.br/ acm/seer/index.php/arquivos/article/view/313/180>. Acesso em: 11 nov. 2017.

BRINATI, L. M. et al. Prevalência e fatores associados à neuropatia periférica em indivíduos com diabetes mellitus. Revista de Pesquisa: Cuidado é Fundamental Online, v. 9, n. 2, p. 347, 2017. Disponível em: <https://pesquisa.bvsalud.org/portal/resource/pt/bde-30363>. Acesso em: 07 nov. 2017.

CAMILA LEANDRA BUENO DE ALMEIDA. Estudo químico e farmacológico de frutos silvestres obtidos de Santa Catarina e Mato Grosso. 2013. Universidade do Vale do Itajaí, 2013. Disponível em: <https://siaiap39.univali.br/repositorio/bitstream/repositorio/1431/1/Camila Leandra Bueno de Almeida.pdf>. Acesso em: 07 nov. 2017.

CIPRIANI, A. Investigação fitoquímica e avaliação da atividade hipoglicemiante, antioxidante, anticolinesterásica e antimicrobiana das folhas de Eugenia involucrata (MYRTACEAE). 2016. 134f. Dissertação (Mestrado em Química). Fundação Universidade Regional de Blumenau. Santa 
Catarina. Curso de Pós-Graduação em Química. Disponível em: < https://www.furb.br/web/3382/ cursos/programa-pos-graduacao/quimica/eventos-e-bancas/banca-de-dissertacao-demestrado-em-quimica/2312>. Acesso em: 09 nov. 2017.

COYLE, J.; Kershaw, P. Galantamine, a Cholinesterase Inhibitor that Allosterically Modulates Nicotinic Receptors: Effects on the Course of Alzheimer's Disease. Biol. Phychiatry, v. 49, p. 289299, 2001. Disponível em: https://revista.facene.com.br/index.php/revistane/article/view/247/156. Acesso em: 14 de jun. 2021.

CUMMINGS, J. L. The role of cholinergic agents in the management of behavioural disturbances in Alzheimer's disease. International Journal of Neuropsychopharmacology, v. 3, n. SUPPL. 2, 2000. Disponível em: <https://pubmed.ncbi.nlm.nih.gov/11343621/>. Acesso em: 09 nov. 2017.

DALLAQUA, B.; DAMASCENO, D. C. Comprovação do efeito antioxidante de plantas medicinais utilizadas no tratamento do diabetes mellitus em animais: Artigo de atualização. Revista Brasileira de Plantas Medicinais, v. 13, n. 3, p. 367-373, 2011. Disponível em: <https://www.scielo.br/j/rbpm/a/ YhmSrXSbtdgdzq3L5TGBYmg/?lang=pt\&format=pdf>. Acesso em: 27 abr. 2021.

DARVESH, S.; HOPKINS, D.A.; GEULA, C. Neurobiology of butyrylcholinesterase. Nature Reviews Neuroscience, v. 4, p. 131-138, 2003. Acesso em: 08 nov. 2017.

DOS SANTOS, P. M. et al. Perfil de flavonoides e avaliação do potencial antioxidante e citotóxico de bauhinia purpurea (fabaceae) da região amazônica. Quimica Nova, v. 37, n. 1, p. 89-94, 2014. Disponível em: <https://www.scielo.br/j/qn/a/zFxLgcZ9ffzd9V48QC4Hgys/?̨format=pdf\&lang=pt>. Acesso em: 27 abr. 2021.

ELLMAN, G. L. et al. A new and rapid colorimetric determination of acetylcholinesterase activity. Biochem Pharmacol., v. 7, p. 88-95, 1961. Disponível em: < https://www.sciencedirect.com/ science/article/abs/pii/0006295261901459>. Acesso em: 09 nov. 2017.

FERREIRA, L. T. et al. Diabetes melito: hiperglicemia crônica e suas complicações. Arquivos Brasileiros de Ciências da Saúde, v. 36, n. 3, p. 182-188, 2011. Disponível em: <https://www. portalnepas.org.br/abcs/article/view/59>. Acesso em: 08 nov. 2017.

FISHMAN, E.B.; SIEKi, G.C.; MaCCALLUM, R.D.; BIRD, E.D.; VOLICER, L.; MARQUI, J.K. Distribution of the molecular forms of acetylcholinesterase in human brain:alterations in dementia of the Alzheimer type. Annals of Neurology, v.19, p. 246-252, 1986. Acesso em: 17 nov. 2017.

FRANCISQUETI, F. V. et al. The role of oxidative stress on the pathophysiology of metabolic syndrome. Revista da Associacao Medica Brasileira, v. 63, n. 1, p. 85-91, 2017. Disponível em: <https://www.scielo.br/scielo.php?script=sci_arttext\&pid=\$0104-42302017000100085\#: :text=Oxidati ve stress plays an important, a mechanism already described above.>. Acesso em: 07 nov. 2017.

GEULA, C. et al. Postnatal development of cortical acetylcholinesterase-rich neurons in the rat brain: permanent and transient patterns. Exp. Neurol. 134, 157-178. 1995. Disponivel em: < https:// wWw.sciencedirect.com/science/article/abs/pii/S0014488685710461>. Acesso em: 06 nov. 2017.

GRAFIUS, M.A.; BOND, H.E.; MILLAR, D.B. 1971. Acetylcholinesterase interaction with alipoprotein matrix. European Journal of Biochemistry, v. 22, p. 382-390, 1971. Acesso em: 10 nov. 2017.

HASHEMPUR, M. H. et al. Complementary and alternative medicine use in Iranian patients with diabetes mellitus. Journal of Integrative Medicine, v. 13, n. 5, p. 319-325, 2015. Disponível em: <https://pubmed.ncbi.nlm.nih.gov/26343103/>. Acesso em: 06 nov. 2017. 
EFEITO DA FRAÇÃo ACETATO DE ETILA EXTRAÍDA DAS FOLHAS DE EUGENIA INVOLUCRATA (MYRTACEAE) SOBRE A ATIVIDADE DA...

INTERNATIONAL DIABETES FEDERATION. IDF Diabetes Atlas [Internet]. 6a ed. Brussels: International Diabetes Federation 2014. Disponível em: <http://www.idf.org/diabetesatlas>. Acesso em: 06 nov. 2017. Acesso em: 07 nov. 2017.

LAW, A.; GAUTHIER, S.; QUIRION, R. Say NO to Alzheimer's disease: The putative links between nitric oxide and dementia of the Alzheimer's type. Brain Research Reviews, v. 35, n. 1, p. 73-96, 2001. Disponível em: <https://pubmed.ncbi.nlm.nih.gov/11245887/>. Acesso em: 07 nov. 2017.

LEHMANN, A. C. et al. Efeitos da fração acetato de etila de Tabernaemontana catharinensis sobre respostas glicêmicas e estresse oxidativo de Rattus norvegicus diabéticos. Nutrire, v. 40, n. 3, p. 297-305, 2015. Disponível em: <http://sban.cloudpainel.com.br/files/revistas_publicacoes/477.pdf>. Acesso em: 09 nov. 2017.

LIMA, C. C. Estudo químico e Avaliação do Potencial Biológico de Extratos e Compostos Isolados de Folhas e Caule de Myrciaria ferruginea O. Berg (Myrtaceae). 2016. 177f.. Tese (Doutorado em Ciências). Instituto de Química e Biotecnologia. Universidade Federal de Alagoas. Programa de Pós-Graduação em Química. Disponível em: <http://www.repositorio.ufal.br/handle/riufal/1599>. Acesso em: 09 nov. 2017.

MAZZANTI, C. M. et al. Extrato da casca de Syzygium cumini no controle da glicemia e estresse oxidativo de ratos normais e diabéticos. Ciência Rural, v. 33, n. 6, p. 1061-1065, 2003. Disponível em: <https://www.scielo.br/scielo.php?script=sci_arttext\&pid=\$0103-84782003000600010\#: :text=0 Syzygium cumini\%2C também conhecido,e diabéticos induzidos por aloxano.>. Acesso em: 11 nov. 2017.

MELO, J.B.; AGOSTINHO, P.; OLIVEIRA, C.R. Involvement of oxidative stress in the enhancement of acetylcholinesterase activity induced by amyloid beta-peptide. Neuroscience Research, v. 45, p. 117-127, 2003. Acesso em: 17 nov. 2017.

MESULAM, M. M. et al. Acetylcholinesterase knockouts establish central cholinergic pathways and can use butyrylcholinesterase to hydrolyze acetylcholine. Neuroscience, v. 110, n. 4, p. 627-639, 2002. Disponível em: <https://pubmed.ncbi.nlm.nih.gov/11934471/>. Acesso em: 11 nov. 2017.

MOREIRA, P. L.; BOAS, P. J. F. V.; FERREIRA, A. L. A. Association between oxidative stress and nutritional status in the elderly. Revista da Associacao Medica Brasileira, $v$. 60, n. 1, p. 75-83, 2014. Disponível em: <https://www.scielo.br/scielo.php?script=sci_ arttext\&pid=S0104-42302014000100075>. Acesso em: 13 nov. 2017.

PETRONILHO, E.; PINTO, ANGELO, C.; VILLAR, J. D. F. ACETILCOLINESTERASE: ALZHEIMER E GUERRA QUÍMICA. Revista Militar de Ciência e Tecnologia. 12 f.. 20110 . Disponível em: < http://rmct.ime. eb.br/arquivos/RMCT_3_tri_2011/RMCT_067_E5A_11.pdf >. Acesso em: 13 nov. 2017.

ROCHA, F. D. et al. Diabetes mellitus e estresse oxidativo: produtos naturais como alvo de novos modelos terapêuticos. Rev. Bras. Farm., 87(2): 49-54, 2006. Disponível em: < https://pt.slideshare.net/ johnwashingtonc/diabetes-mellitus-ok-44005816>. Acesso em: 11 nov. 2017.

RODRIGUES DA SILVA, L. et al. Flavonóides: constituição química, ações medicinais e potencial tóxico. Acta toxicológica argentina, v. 23, n. 1, p. 36-43, 2015. Disponível em: <https://www. researchgate.net/publication/317539623_Flavonoides_constituicao_quimica_acoes_medicinais_e_ potencial_toxico>. Acesso em: 15 nov. 2017.

SCHALKWIJK, C. G.; STEHOUWER, C. D. A. Vascular complications in diabetes mellitus: The role of endothelial dysfunction. Clinical Science, v. 109, n. 2, p. 143-159, 2005. Disponível em: <https:// pubmed.ncbi.nlm.nih.gov/16033329/>. Acesso em: 17 nov. 2017. 
SERON, V. D.; FURLAN, M. M. D. P. Papel do Chá Verde e seus Componentes no Tratamento do Diabetes Mellitus Tipo 2. Saúde e Pesquisa, v. 3, n. 3, p. 379-383, 2010. Disponível em: <https:// periodicos.unicesumar.edu.br/index.php/saudpesq/article/view/1523\#: :text=O chá verde\%2C conhecido por,qualidade de vida dos indivíduos.>. Acesso em: 15 nov. 2017.

SILVA, N. R. da; COSTA, C. E. M. da. A hiperglicemia e os mecanismos envolvidos nas disfunções. Arq. Ciênc. Saúde Unipar, Umuarama, v. 12, n. 3, p. 265-270, 2008. Disponível em: <https://revistas. unipar.br/index.php/saude/article/view/2544/1987>. Acesso em: 08 nov. 2017.

SILVA, S. R. S.; OLIVEIRA, T. T.; NAGEM, T. J. Uso do chá preto (Camellia sinensis) no controle do diabetes mellitus. Revista de Ciencias Farmaceuticas Basica e Aplicada, v. 31, n. 3, p. 133-142, 2010. Disponível em: <https://rcfba.fcfar.unesp.br/index.php/ojs/article/view/358\#: :text=Os hábitos alimentares exercem um,o diabetes\%2C e suas complicações.>. Acesso em: 13 nov. 2017.

\section{SILVEIRA, S. et al. ATIVIDADE ANTICOLINESTERÁSICA DOS FRUTOS DE Myrcianthes pungens (O.BERG)}

D.LEGRAND (MYRTACEAE). Arq. Ciênc. Saúde UNIPAR, Umuarama, v. 15, n. 2, p. 127-133, 2011. Disponível em: < https://www.revistas.unipar.br/index.php/saude/article/view/3707>. Acesso em: 13 nov. 2017.

TSAKIRIS, S.; ANGELOGIANNI, P.; SCHULPIS, K.H.; STAVRIDIS, J.C. Protective effect of L-phenylalanine on rat brain acetylcholinesterase inhibiton induced by free radicals. Clinical Biochemistry, v. 33, p. 103-106, 2000. Acesso em: 17 nov. 2017.

WILD, S. et al. Global Prevalence of Diabetes: Estimates for the year 2000 and projections for 2030. Diabetes Care, v. 27, n. 5, p. 1047-1053, 2004. Disponível em: <https://pubmed.ncbi.nlm.nih. gov/15111519/>. Acesso em: 09 nov. 2017. 